\title{
Guest Editorial: new developments in ambient information systems
}

\author{
Ansar-Ul-Haque Yasar $^{1} \cdot{\text { Zahoor } \text { Khan }^{2} \cdot \text { Elhadi M. Shakshuki }}^{3}$
}

Received: 10 November 2014/ Accepted: 15 January 2015/Published online: 15 March 2015

(C) Springer-Verlag Berlin Heidelberg 2015

This special issue is based on the best papers from the 5 th International Conference on Ambient Systems, Networks and Technologies (ANT), which was held at Hasselt, Belgium, on 2-5 June 2014. The conference attracted a large number of scientific papers that contributed to the state-of-the-art in the areas of pervasive and ambient information systems. In the 5th edition of the conference there was a special focus on real-life mobility issue from the intelligent and agent-based transportation domain. All the papers selected for this special issue have been extended from their original versions and underwent three rounds of rigorous peer-review process. Based on the reviewers' feedback, as well as the evaluations of the Guest Editors, six papers were selected for this special issue from nine invited submissions. The accepted papers cover interesting works on new developments in ambient and networked systems. The accepted papers cover interesting works on new developments in ambient and networked systems such as smart grids, intelligent mobility, agentbased simulations, vehicular networks and systems security.

Elhadi M. Shakshuki

elhadi.shakshuki@acadiau.ca

Ansar-Ul-Haque Yasar

ansar.yasar@uhasselt.be

Zahoor Khan

Zahoor.Khan@hct.ac.ae

1 Transportation Research Institute, Hasselt University, Hasselt, Belgium

2 Computer Information Science, Higher Colleges of Technology, Fujairah, UAE

3 Jodrey School of Computer Science, Acadia University, Wolfville, Canada
The first paper by Fenu and Pau is entitled "Evaluating complex network indices for vulnerability analysis of a territorial power grid". The authors applied optimization strategies on power flows, and verify the current degree of self-sufficiency of the network, with special reference to the effect of natural variations in wind-based production. Then, proposed a method to assess collateral damage to the network resulting from a localized failure, and proceed to perform complex network analysis on multiple instances of the network, looking for correlations between estimated damages and between centrality indices, with the purpose of attempting to determine which model is best suited to predict features of our network.

The second paper by Marey et al. is entitled "Decision making under subjective uncertainty in argumentation-based agent negotiation". This paper provided a study and analyses of agents' uncertainty in Argumentation-Based Agent Negotiation (ABAN), and presented two methods. The first method extends a previously defined approach for measuring agents' uncertainty based on Shannon entropy. The second method introduces a new technique to assess agents' uncertainty using hypothesis testing. The objective is to build a comprehensive framework for tackling agents' uncertainty in ABAN. Furthermore, they proposed a Markov Decision Process (MDP)based framework to incorporate the different situations of agents' uncertainty based on the available arguments or offers. Besides, they discussed the uncertainty issues in two special cases based on the different classes that arguments can belong to. In addition to the theoretical analysis of arguments uncertainty, they discussed the implementation of the proposed approach by applying it to a concrete case study (Buyer/Seller) scenario. The obtained empirical results confirm the effectiveness of using their uncertainty-aware techniques and show that their negotiating agents outperform others that use pure argumentation with no uncertainty consideration. 
The third paper by Boukharrou et al. "Dealing with temporal failure in ambient systems: A dynamic revision of plans" presented AgLOTOS as an algebraic language dedicated to the specification of agent plans in ambient systems taking into account timing constraints. They showed how a plan is built automatically as a system of concurrent processes from the Belief-Desire-Intention (BDI) attitudes of the agent. The AgLOTOS semantics allows dealing with possible plan execution failures, caused by the passing of time performance of actions. In this situation, the information is captured at the mental process level allowing updating the agent mental state. Moreover, the associated semantics accords with the possibility of revising the agent plan, as the set of intentions changes.

The fourth paper by Lutzenberger et al. is entitled "A common approach to intelligent energy and mobility services in a smart city environment". The authors of this paper presented their gained experiences during the last years and presented the developed solutions that consider energy and mobility-related problems jointly. Due to the fact that electric vehicles have not broadly entered the vehicle market there are many attempts to convince producers to integrate technologies that utilise embedded batteries for purposes different from driving. The vehicleto-grid technology, for instance, literally turns electric vehicles into a mobile battery, enabling new areas of applications (e.g. to provide regulatory energy, to do gridload balancing, or to buffer surpluses of energy) and business perspectives. Utilising a vehicle's battery; however is not without a price-in this case: the driver's mobility. Given this dependency, it is interesting that most available works consider the application of electric vehicles for energy and grid-related problems in isolation, that is, detached from mobility-related issues. The authors are members of the DAI-Lab that has engaged in a large number of past and upcoming projects concerned with two aspects of managing electric vehicles, namely: energy and mobility.
The fifth paper by Feukeu et al. "Performance evaluation of the ASDA in a vehicular network: MAC approach in IEEE 802.11p" presents a thorough analysis of Automatic Doppler Shift Adaptation (ASSA) method is explored and the strength of the refined ADSA method is evaluated in terms of throughput, elapsed time, packet loss, model efficiency and data transfer rate. These metrics are used to perform a comparative analysis of ADSA versus Adaptive Modulation Code (AMC) and Auto-Rate Fallback (ARF). Results from the analysis shows that the ADSA approach is very effective and has a strong robustness compared to ARF and AMC with up to 300-700 \% improvement in throughput and a 60-75\% reduction in consumed time.

The sixth and last paper by Ouchani et al. "Generating attacks in SysML activity diagrams by detecting attack surfaces" developed a practical framework that automates the development process of a secure system which is essential to detect as early as possible the system's vulnerable points. The authors formalized a system as SysML activity diagrams and in the same formalism they modelled libraries of patterns taken from standard catalogues of social engineering and technical attacks. An algorithm that they defined, navigate the system's diagrams in search for its attack surfaces; then, it evaluates the possibility and the probability that the detected weak points host attacks among those in the modelled library. The authors proved the correctness and the completeness of their proposed approach and demonstrated how it works on a use case scenario.

The guest editors would like to take this opportunity to thank all the authors for the efforts they put in the preparation of their manuscripts and for their valuable contributions. We wish to express our deepest gratitude to the referees who provided very useful and thoughtful feedback to our authors. Our sincere thanks go to the Editor-in-Chief for his kind help and support. 